\title{
Incidentally detected odontoma within a dentigerous cyst
}

\author{
Kwang Seog Kim, \\ Han Gyeol Lee, \\ Jae Ha Hwang, \\ Sam Yong Lee
}

Department of Plastic and Reconstructive Surgery, Chonnam National University Medical School, Gwangju, Korea

\begin{abstract}
Odontoma is an asymptomatic slow-growing odontogenic tumor. It is usually found by chance in the maxilla or mandible on radiography, or when it deforms the adjacent teeth. It is commonly found in patients who are 30 years of age or younger. We report our encounter with an odontoma within a dentigerous cyst found incidentally in a 56-year-old man. He presented with abnormal fullness in the right infraorbital area of the cheek. During the evaluation of the mass, we incidentally detected the odontogenic tumor within a dentigerous cyst in the patient's maxilla. Under general anesthesia, complete surgical drainage of the infraorbital cystic mass was performed. Enucleation of the odontogenic tumor and a bone grafting from the iliac bone were also performed. The final diagnosis was odontoma. After 2 years of follow-up, there was no sign of recurrence of the tumor. In case of odontogenic tumors, even in old patients, it is important to suspect an odontoma. When odontoma accompanies a dentigerous cyst, surgical excisional biopsy should be performed to rule out malignancy. In case of a large bony defect after enucleation, autogenous bone grafting is essential for reconstruction.
\end{abstract}

Keywords: Alveolar bone grafting / Dentigerous cyst / Odontoma / Surgical procedure

\section{INTRODUCTION}

Odontogenic tumors include ameloblastoma, keratocystic odontogenic tumor, adenomatoid odontogenic tumor, odontoma, calcifying cystic odontogenic tumor, myxoma, ameloblastic carcinoma, and primary interosseous squamous cell carcinoma, among others [1]. Odontoma is one type of odontogenic tumors. It grows slowly, is asymptomatic, and is usually found in patients who have been treated for other oral conditions. It is commonly detected in patients who are 30 years of age or younger [2]. However, if not treated adequately, it can result in deformity of the adjacent teeth, fractures, or any other damage to the jaw [3]. It may also cause carcinomatous transformation

\section{Correspondence: Kwang Seog Kim}

Department of Plastic and Reconstructive Surgery, Chonnam National University

Medical School, 42 Jebong-ro, Dong-gu, Gwangju 61469, Korea

E-mail: pskim@chonnam.ac.kr

Received January 1, 2018 / Revised June 1, 2018 / Accepted September 1, 2018 according to its clinical characteristics. Hence, it is very important to treat odontomas. Here, we report a case of a 56 -year-old man with an incidentally detected odontoma.

\section{CASE REPORT}

A 56-year-old man presented with a chief complaint of a growing mass on the right infraorbital area on the cheek. It was soft and painless with a non-tender swelling. There was a history of trauma on the lesion 23 years ago. Computed tomography (CT) images revealed a well-defined, thin-walled, non-enhancing, multilobulated cystic lesion of $3.4 \times 2.2 \times 3-\mathrm{cm}$ dimensions in the right infraorbital area. The lesion was clinically diagnosed as a mucocele. While evaluating the infraorbital mucocele on CT, we unintentionally detected another mass. There was an unerupted tooth in the mid-portion of the hard palate of dimensions: $1.3 \times 1.1 \times 1.2 \mathrm{~cm}$, observed within the dentigerous 

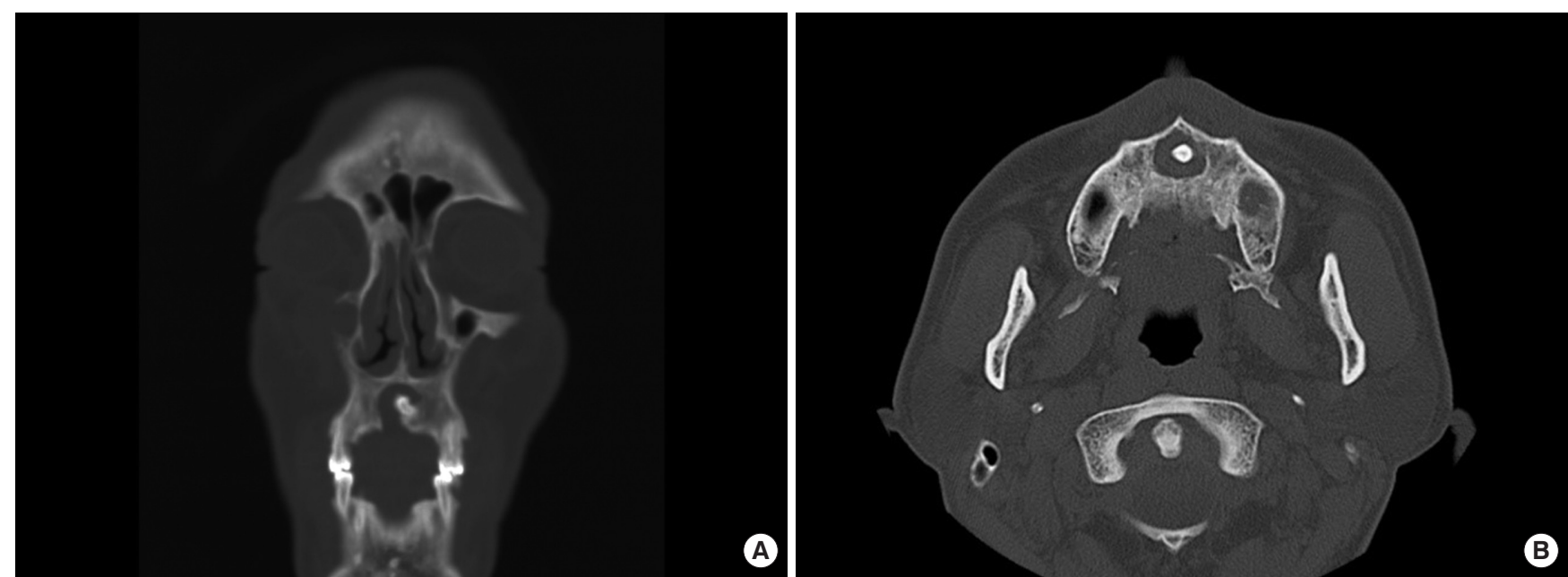

Fig. 1. Facial computed tomography showed unerupted tooth of $1.3 \times 1.1 \times 1.2 \mathrm{~cm}$ size within a dentigerous cyst. (A) Coronal view. (B) Axial view.
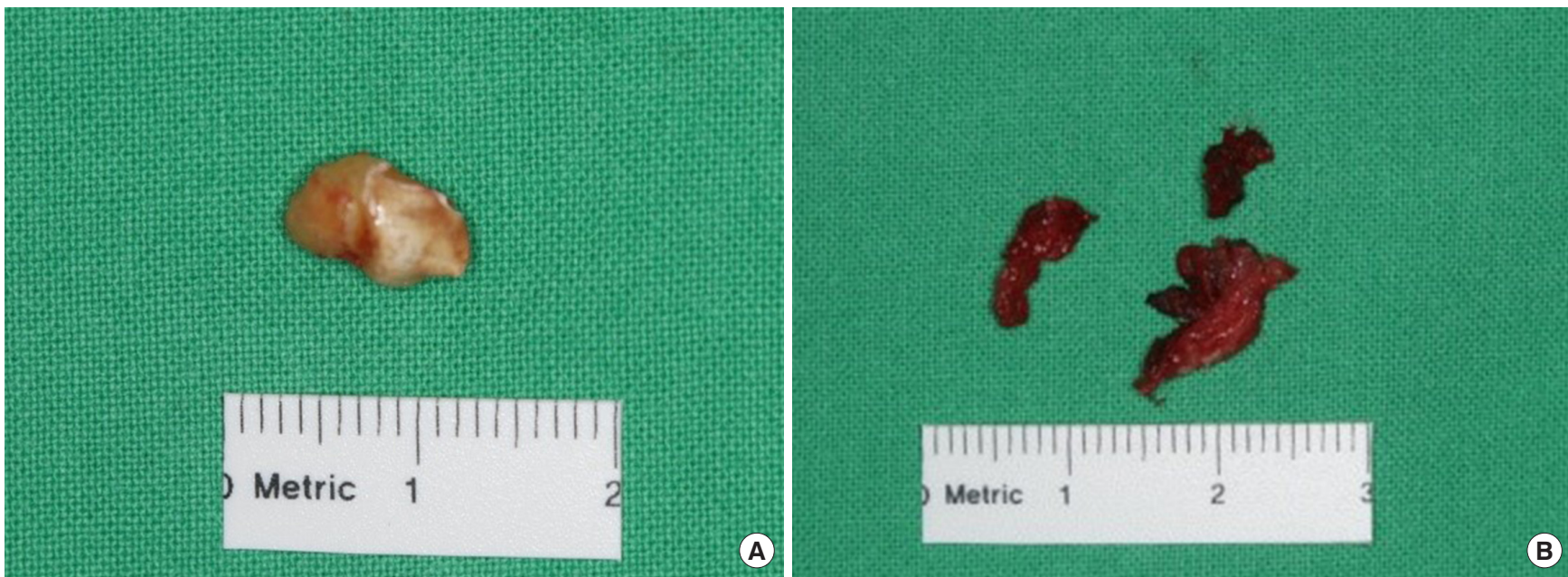

Fig. 2. After removal of the cystic component, an unerupted tooth measuring $1 \mathrm{~cm}(\mathrm{~A})$ and a dentigerous cyst (B) were observed.

cyst (Fig. 1). This was suspected to be an odontogenic tumor. The patient did not present signs of diplopia, or any tooth pathology.

Complete surgical drainage of the right infraorbital mucocele and enucleation of the odontogenic tumor were performed under general anesthesia (Fig. 2). However, when we enucleated the odontogenic tumor, a bony defect of $2 \times 2 \mathrm{~cm}$ size was observed in the anterior palate (Fig. 3). Autogenous bone grafting from the right iliac crest was performed to reconstruct the defect.

The results from the histopathological examination revealed a structure resembling a single-rooted tooth (Fig. 4). The final diagnosis given was compound odontoma. There was no connection between the infraorbital mucocele and the maxillary odontoma. After 2 years of follow-up, there were no signs of complications nor recurrence of the tumor. We obtained the patient's medical records and reviewed the related literature.

\section{DISCUSSION}

Odontoma is a generally an asymptomatic lesion that is a casual finding in the course of routine radiologic studies. It is formed of enamel, dentin, cementum, and pulp tissue [2]. There are two types of odontomas according to the latest classification of the World Health Organization [1]. The compound odontoma is a radiopaque mass composed of multiple small calcified structures that are anatomically similar to normal teeth and are typically surrounded by a narrow radiolucent zone on radiography [2,4-6]. The complex odontoma is composed of an amorphous mass of calcified material that exhibits a radiodensity similar to that of teeth, and is surrounded by a narrow radiolucent rim on the radiograph. However, it does not anatomically 


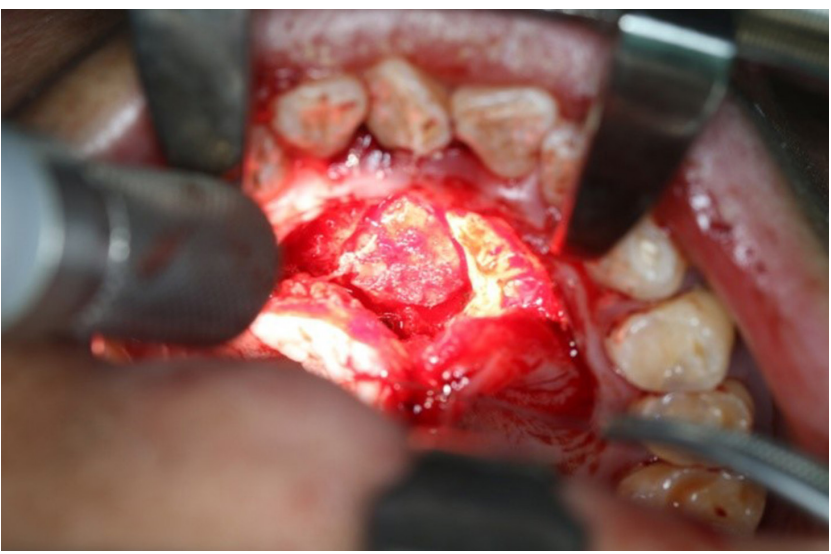

Fig. 3. After enucleation, a bony defect of $2 \times 2 \mathrm{~cm}$ remained.

resemble teeth $[2,7]$. In our case, a structure resembling a single-rooted tooth was observed on histopathological examination that aided in the diagnosis of compound type odontoma.

Clinically, odontomas are either intraosseous or extraosseous. The intraosseous odontomas occur inside the maxilla or mandible, and may eventually erupt into the oral cavity. Peripheral odontomas arising from the extraosseous soft tissues are rare and have a tendency to exfoliate. Our patient had an intraosseous odontoma that did not erupt into the oral cavity [8].

Most odontomas occur in the first and second decades of life, especially among female patients. Odontomas are typically involved in delayed tooth eruption; thus, in children or adolescents with dental anomalies or jaw deformation, the possibility of odontoma must be investigated by radiography [2]. The diagnosis is usually based on routine radiography findings. Additional evaluation with $\mathrm{CT}$ is necessary to determine the type, features, and extension of the odontoma. In our case, the patient was 56 years of age and did not exhibit any dental anomaly or jaw deformation. The case substantially differs from the known characteristics of odontoma with the exception of incidental detection.

Patients with odontoma do not usually exhibit symptoms, but odontoma may be found within cysts. The dentigerous cyst arises due to the accumulation of fluid between reduced enamel epithelium and crown of an unerupted tooth [9]. In these cases, it may cause displacement of erupting teeth or dysphagia, and may also lead to carcinomatous transformation.

Normal odontomas are treated using conservative surgical enucleation, depending on the size and dentition problems. If not treated adequately, odontomas can lead to cyst formation and conversion to malignancies such as odontoameloblastoma. It is important to confirm the pathological diagnosis because ameloblastic odontoma and ameloblastic fibro-odontoma resemble common odontomas.

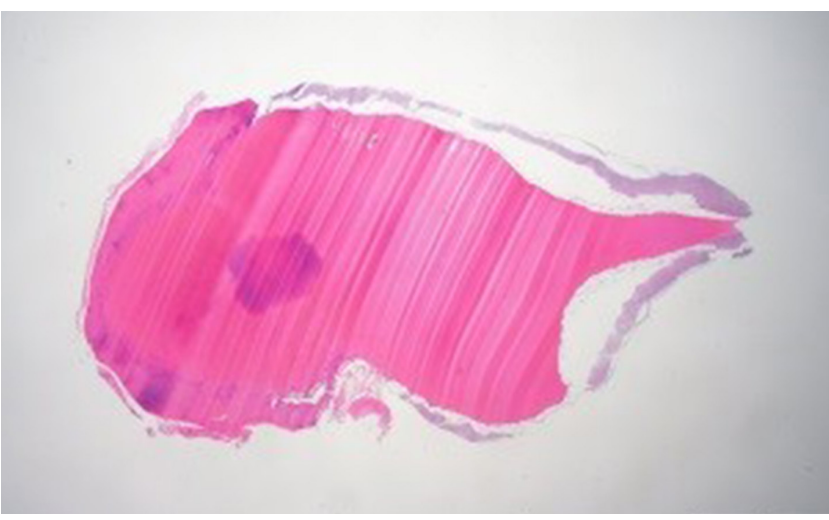

Fig. 4. Pathological examination of the specimen. A tooth-like structure is observed $(\mathrm{H} \& \mathrm{E}, \times 40)$.

On the other hand, the treatment of odontoma within a dentigerous cyst is very different. The most important factor in treatment is the age of the patient. There is no study reporting the exact age and the appropriate treatment method. In general, odontomas within dentigerous cysts in young children are less aggressive than those in adults, and therefore, marsupialization is preferred [9]. If the patient is older, an odontoma within a dentigerous cyst is thought to be aggressive, leading to significant jaw destruction, and requiring enucleation. In our case, the patient had no symptoms. However, the odontoma accompanying the dentigerous cyst carried the risk of jaw destruction or possible carcinomatous transformation. Hence, the patient was treated with enucleation.

Bony defects can lead to subsequent fractures or damage to the jaw. In our case, there was a $2 \times 2-\mathrm{cm}$-sized bony defect in the alveolar ridge after enucleation of the odontoma. Hence, we performed autogenous bone grafting from the iliac crest to reconstruct both compact and cancellous bony defects of the hard palate. The results were satisfactory, and the contour of the alveolar ridge was well maintained. There was low donor site morbidity.

The implications of our findings, however, are limited by the fact that we analyzed only one case. If we had more cases, we could have studied the correlation of the size of the odontoma with age and treatment. Further studies and long-term data are required to investigate these relationships.

In conclusion, when surgeons encounter a patient with an odontogenic tumor, even if the patient is aged, it is important to suspect an odontoma. If the odontoma accompanies a dentigerous cyst, it can lead to carcinomatous transformation; thus, marsupialization or enucleation will be required. In addition, when a large bony defect is observed after excision of the odontoma, autogenous bone grafting is essential to reconstruct the defect. 


\section{NOTES}

\section{Conflict of interest}

KSK, an editor-in-chief of the Archives of Craniofacial Surgery, is the corresponding author of this article. However, he played no role whatsoever in the editorial evaluation of this article or the decision to publish it. Except for that, no potential conflict of interest relevant to this article was reported.

\section{Ethical approval}

The study was approved for exemption from Institutional Review Board review by the IRB of Chonnam National University Hospital (CNUH-EXP-2018-229) and performed in accordance with the principles of the Declaration of Helsinki.

\section{Patient consent}

The patient provided written informed consent for the publication and the use of his images.

\section{ORCID}

Kwang Seog Kim https://orcid.org/0000-0002-6766-4640

Han Gyeol Lee https://orcid.org/0000-0001-7835-0172

Jae Ha Hwang https://orcid.org/0000-0001-7556-3594

Sam Yong Lee https://orcid.org/0000-0002-3185-2519

\section{REFERENCES}

1. da Silva LP, de Paiva Macedo RA, Serpa MS, Sobral AP, de
Souza LB. Global frequency of benign and malignant odontogenic tumors according to the $2005 \mathrm{WHO}$ classification. J Oral Diagn 2017;2:e20170044.

2. Boffano P, Zavattero E, Roccia F, Gallesio C. Complex and compound odontomas. J Craniofac Surg 2012;23:685-8.

3. Zerener T, Sencimen M, Bayar GR, Kan B, Ayberk H, Arici G. Immediate reconstruction using autogenous bone graft in huge compound odontoma. Oral Health Dent Manag 2015;15: 243-6.

4. Pacifici A, Carbone D, Marini R, Pacifici L. Surgical management of compound odontoma associated with unerupted tooth. Case Rep Dent 2015;2015:902618.

5. John JB, John RR, Punithavathy I, Elango I. Compound odontoma associated with maxillary primary tooth: a case report. JIADS 2010;1:49-51.

6. de Oliveira BH, Campos V, Marcal S. Compound odontoma: diagnosis and treatment: three case reports. Pediatr Dent 2001; 23:151-7.

7. Pillai A, Moghe S, Gupta MK, Pathak A. A complex odontoma of the anterior maxilla associated with an erupting canine. BMJ Case Rep 2013;2013:bcr2013200684.

8. Shamaskin RG, Svirsky JA, Kaugars GE. Intraosseous and extraosseous calcifying odontogenic cyst (Gorlin cyst). J Oral Maxillofac Surg 1989;47:562-5.

9. Jayam C, Bandlapalli A, Patel N, Choudhary RS. A case of impacted central incisor due to dentigerous cyst associated with impacted compound odontome. BMJ Case Rep 2014;2014: bcr2013202447. 